\title{
La competencia emocional en personas con sordera
}

\author{
The emotional competence in people with deafness \\ Competência emocional em pessoas com surdez
}

\begin{abstract}
Carla Díaz Salazar Iglesias
Magister en trastornos del lenguaje

Maestra de audición y lenguaje de la institución pública CEIP Ramón y Cajal de Vigo

(España)

carladiazsalazar@gmail.com
\end{abstract}

\begin{abstract}
Resumen
La presente revisión pretende desarrollar un acercamiento hacia el conocimiento del mundo emocional que experimenta el alumnado con sordera (con o sin implante coclear), fundamentalmente en aquellos aspectos relativos a la competencia emocional, como resultan la identificación, gestión, comprensión y expresión de emociones. A lo largo del presente trabajo se analizarán diez estudios llevados a cabo en diferentes partes del mundo que abordan esta temática, junto con aportaciones de otras investigaciones o autores que guardan relación con la misma, desarrollando a su vez una comparación con el alumnado oyente en lo que se refiere a dichas competencias. Asimismo, se profundiza en la necesidad de desarrollar estas habilidades emocionales en la escuela y en este colectivo especialmente, recordando que el desarrollo armónico de cada ser constituye una intrínseca relación con el ámbito emotivo que lo conforma.
\end{abstract}

Palabras clave: Discapacidad auditiva, alfabetización emocional, sordera, competencia emocional, Teoría de la mente.

\begin{abstract}
The present review aims to develop an approach to the knowledge of the emotional world that is experiencing the pupils with deafness (with or without cochlear implant), mainly in those aspects related to the emotional competence, as are the identification, management, understanding and expression of emotions. Throughout this work is analyzed ten studies carried out in different parts of the world that deal with this subject, together with contributions from other research or authors that are related to the same, developing to turn a comparison with the students listener in regard to these competencies. It is also deepened in the need to develop these emotional skills in the school and in this collective especially, recalling that the harmonious development of every being is an intrinsic relationship with the field of emotion that conforms him.
\end{abstract}

Key words: Hearing loss, emotional alphabetization, deafness, emotional competence, Theory of the Mind. 


\section{Resumo}

A presente revisão pretende desenvolver uma abordagem ao conhecimento do mundo emocional experimentado por estudantes com surdez (com ou sem implante coclear), principalmente nos aspectos relacionados à competência emocional, como identificação de resultado, gerenciamento, compreensão e expressão de emoções. Ao longo do presente trabalho, serão analisados dez estudos realizados em diferentes partes do mundo que abordam esse tema, juntamente com contribuições de outras pesquisas ou autores que estão relacionados a ele, desenvolvendo, por sua vez, uma comparação com os alunos da audiência no que se refere a essas competências. Da mesma forma, aprofunda a necessidade de desenvolver essas habilidades emocionais na escola e especialmente neste grupo, lembrando que o desenvolvimento harmônico de cada ser constitui uma relação intrínseca com a esfera emocional que o molda.

Palavras-chave: deficiência auditiva, alfabetização emocional, surdez, competência emocional, teoria da mente.

\section{Introducción}

Según Díaz (2013) se conoce que toda información independientemente del ámbito al que se refiera, tiene siempre una perspectiva y una vertiente relacionada con el ámbito social, en la medida que afecta a la sociedad o parte de la misma y a las personas que las conforman. Además, siguiendo a López (2007) desde un enfoque ético, la investigación constituye un agente que contribuirá a mejorar la realidad social, especialmente en aquellos colectivos de personas que se encuentren en una situación de desventaja, y cabe considerar que la sordera supone una desventaja bio-evolutiva, además de social. Esto constituye según Gutiérrez (2007) un inconveniente causado por dos aspectos: en primera instancia, el de encontrarte en medio de una sociedad conformada mayoritariamente por personas oyente-parlantes y diseñada desde esos parámetros, y en segundo lugar a causa de los obstáculos derivados de no poder utilizar el sentido del oído, el cual funciona multidireccionalmente y de manera simultánea con la vista.

De hecho, la historia educativa de las personas que padecen sordera muestra que han sido apartadas y discriminadas de la sociedad por ser diferentes, y han tenido que luchar de manera incansable, para lograr ser respetadas y reconocidas como integrantes de una comunidad con lengua propia; la lengua de signos, la cual les otorga una identidad. Tenemos una muestra de ello, como apunta Benvenuto (2009) remontándonos al siglo VI, Italia, donde las personas con sordera eran acogidas por congregaciones católicas que se regían por la regla del silencio de Saint Benoît, la cual consistía en comunicar mediante signos sus necesidades cotidianas por parte de los monjes.

Sin embargo, entre finales de los años noventa y los primeros del siglo XXI, se ha producido un interés por analizar la influencia de las emociones en el ámbito de la Inteligencia Emocional (de ahora en adelante, IE) desde que Mayer y Salovey, en la década de los 90, continuando con la tendencia iniciada por otros grandes psicólogos como Wechsler (1940), Gardner (1940) o Sternberg (1988), desarrollaron por primera vez la IE y la posterior difusión de Goleman con su libro, en 1995 (Ramos, Recondo y Enríquez 2012). 
A raíz de esta revolución emocional, la literatura científica ha registrado un número elevado de estudios sobre la IE en sujetos oyentes pero en el ámbito de los/as individuos con sordera el número resulta inferior, pese a que, en los últimos años, se ha ido incrementando el interés por analizar este campo en escolares con discapacidad auditiva (de aquí en adelante, DA).

La IE hace referencia a los procesos intrínsecamente relacionados con el reconocimiento, comprensión, empleo y manejo de los diferentes estados emocionales que experimenta una persona y aquellas que la rodean, para resolver conflictos y regular la conducta (Salovey, 2007). De este constructo de IE se deriva, además, el desarrollo de competencias emocionales que constituyen la suma de conocimientos, capacidades, habilidades y actitudes imprescindibles para comprender, expresar y regular de forma óptima los fenómenos emocionales (Bisquerra, 2003). Dentro de las mismas, las habilidades más estudiadas en este ámbito son la identificación, comprensión y regulación de las emociones (Denham et.al, 2003).

Por otro lado, los diferentes estudios e investigaciones sobre la educación de sujetos con DA se han centrado en aspectos intelectuales y cognitivos, dejando al margen, como afirma Valmaseda (2009), aquellos que se refieren al desarrollo socio-emocional, excluyendo de ese conjunto de objetivos educativos que conforman lo que podría denominarse el "núcleo duro" de las determinaciones educativas respecto al alumnado con sordera, dado que la cultura occidental, hasta el momento, ha otorgado una mayor importancia a los aspectos relacionados con la razón más que en cualquier otra dimensión. Siguiendo esta línea, como apunta Boix (2007) el ser humano se centra mayoritariamente en aquello que piensa, prestando una menor atención a todo el mundo emocional en el que se encuentra sumergido. Así pues, como apuntillaba el crítico de rock Lester Bangs "lo único que vale la pena preguntarse hoy es si la humanidad va a sentir emociones mañana, y qué calidad de vida habrá si la respuesta es no" (LeDoux, 1999, p.25).

Afortunadamente, como hemos visto, la educación de este siglo está cambiando, y parece que quiere caminar de la mano de las emociones, y formar parte del campo de estudio de los investigadores. De hecho, Hindley (2000; citado en Valmaseda, 2009) afirma que la atención de los ámbitos sociales, personales y emocionales del alumnado, resulta de gran interés y preocupación para una gran parte de investigadores $y$ educadores que trabajan con alumnos/as con sordera, ya que muchos de ellos pueden experimentar mayores dificultades en alcanzar un desarrollo armónico de su "yo" más personal y un mayor número de probabilidades de padecer desajustes personales, sociales y emocionales en su etapa adulta.

Por este motivo, la presente investigación ha centrado su foco de interés en analizar el mundo emocional de los estudiantes con DA debido a que la literatura científica que abarca ambos conceptos resulta escasa y/o no es lo suficientemente exhaustiva en los resultados, como se verá a lo largo de esta revisión.

Con todo ello, se desarrolla una revisión acerca de la competencia emocional de los escolares con sordera, con o sin implante coclear (de aquí en adelante IC), en especial aquellos aspectos intrínsecamente relacionados con la identificación, expresión, comprensión de emociones y regulación de sentimientos con el objeto de conocer, 
más en profundidad, su situación emocional y comprobar, a la vez, en qué medida se diferencian de los/as escolares oyentes.

\section{La inteligencia emocional en los escolares con sordera}

Los órganos sensoriales proporcionan informaciones valiosas que inciden en el adecuado desarrollo evolutivo de las personas. El oído, en este caso, permite al ser humano estar en contacto continuo con la realidad y recibir, al mismo tiempo, una ingente cantidad de información sonora. De hecho, antes de nacer $\mathrm{y}$ en los últimos meses de embarazo, la persona oyente ya escucha la voz de su madre y convive, además, con los ruidos, los sonidos externos y las voces (Pigliacampo, 2007). De este modo, el bebé oyente a medida que se desarrolla en su contexto comienza a comprender que éste se conforma de sonidos, palabras, ruidos... y que las personas se comprenden a través de palabras sonoras.

Además, la función auditiva es básica para el desarrollo integral del ser humano y para la conquista del lenguaje. De hecho, Pabón (2009) afirma que, gracias a éste, resulta posible exteriorizar aquello que sentimos y/o pensamos. El lenguaje organiza además la claridad de los pensamientos ordenándolos gramatical, sintácticamente y manera lógica, lo que permite un aumento de la comprensión y expresión de conceptos con diferentes grados de abstracción.

Todo ello aplicado al ámbito de la DA tiene importantes connotaciones puesto que en virtud de su limitación sensorial, las personas con sordera sustituyen con el sentido de la vista y la gestualidad lo que les niega el oído, desarrollando así, una manera colectiva de sentir, de ver el mundo y de actuar (Simón, 2007).
Además, como señala Cañizares (2015) padecer sordera en una sociedad mayoritariamente oyente conlleva problemas como la adquisición espontánea del lenguaje oral o desarrollarse personalmente con una autonomía plena e igualdad de condiciones que una normo-oyente. Esto es, una de las principales diferencias que radica en que una persona sorda tiene notables limitaciones para comunicarse con los demás, y esas dificultades de comunicación se manifestarán en efectos sustanciales a lo largo de sus vidas, desarrollando a la par, un sentimiento de inadaptación, aislamiento social y soledad (Bar-on, 2006).

Pero, es más, la literatura científica disponible sugiere que estos individuos/as tienen más problemas en su desarrollo psicológico, derivados de las dificultades de comunicación, sentimiento de inadaptación, mala autoestima, los cuales afectarán al desarrollo cognitivo, social y emocional, dado que todos se encuentran íntimamente ligados. De hecho, y poniendo el foco de atención en este último, los datos disponibles muestran que los niños/as con sordera tienen, con frecuencia, más problemas emocionales que los oyentes (Meadow, 1980, citado por Hintermair, 2007).

En referencia a la identificación y comprensión de las emociones hay que tener en cuenta que ambos aspectos constituyen procesos graduales que empiezan en el primer año de vida cuando los infantes se hacen sensibles a la importancia emocional de expresiones faciales $y$ entonaciones vocales (Flavell, 2004). A los dos años, empiezan a identificar y etiquetar emociones simples (Denham et al., 2003) es decir, emociones básicas, no 
aprendidas y que duran lo indispensable para cumplir su misión. Dolor, miedo, tristeza, ira y alegría son algunas de las más importantes (Riso, 2012), siendo alrededor de los tres años de edad capaces de expresar emociones verbalmente. En torno a los nueve años, comienzan a apreciar la posibilidad de tener emociones simultáneas en relación con la misma situación. Y dos años después, sobre los 11 años, empiezan a apreciar la concurrencia de las emociones simultáneas con valencia opuesta, como, a modo de ejemplo, la ira y la felicidad (Denham et al., 2003).

Así pues, los niños/as oyentes no sólo observan las expresiones faciales de sus iguales, sino que también escuchan a los/as demás para comprender como dichas expresiones son interpretadas (Rieffe y Terwogt, 2006). Se entiende así, cómo el papel del sonido resulta sumamente vital para provocar y transmitir emociones ya que, sirviéndose de las características del tono, intensidad y ritmo, los/as sujetos aprenden a distinguir la ternura, el enfado, el afecto, etc.

No obstante, también, les permite recibir de forma espontánea experiencias emocionales de sus familias o iguales (Fehr y Exline, 1987). En referencia a estos últimos, Keltner y Gross (1999) aportan, además, que el juego entre niños/as es una plataforma perfecta para practicar las habilidades sociales y para aprender a regular emociones. Así, los infantes aprenden a recoger las señales de su entorno para formar una concepción de cómo deberían comportarse y qué, cómo o cuándo expresar emociones. Evidentemente, cuando se habla de personas con sordera este panorama puede cambiar radicalmente, puesto que las mismas se encuentran con reducidas oportunidades de interactuar con sus familiares o compañeros/as oyentes, debido, en buena parte, a la falta de dominio de un código de comunicación común para ambos. Si bien, esto resulta diferente cuando estamos ante niños/as cuyos padres/madres comparten el mismo déficit, dado que en este caso si compartirían un código común, la lengua de signos.

En este sentido, Farrugia (1982) manifiesta que las interacciones entre padres/madres oyentes e hijos/as con sordera resultan a menudo de menor calidad y cantidad en comparación con la de padres/madres e hijos/as oyentes. Idea apoyada, además, por Peterson y Siegal (2000) que afirman que padres/madres oyentes de niños con sordera tienen dificultades para dialogar sobre asuntos abstractos. Esto incluye conversaciones sobre emociones, ayudar a la hora de etiquetar y hablar como comunicar las mismas de manera apropiada. Asimismo, resaltar que en la actualidad los avances de los implantes cocleares posibilitan aprovechar la audición residual de niños/as y mejorar notablemente su audición, pero todavía existen familias reacias a la colocación de los mismos/as sobre todo en aquellos casos en los que sus padres/madres sufren la misma discapacidad.

Por consiguiente, la insuficiente información a la que se ven sometidos, el quedar reducidos/as a explicaciones incompletas, el no comprender las opiniones y matizaciones de quienes les rodean, contribuye al desarrollo de personalidades simples, inmaduras, egocéntricas y con un alto grado de impulsividad, complicando la comprensión e interiorización de reglas y/o valores (Quirin y Lane, 2012).

Por otra parte, la interacción social menos productiva que sufre el niño/a con sordera y padres oyentes, como consecuencia de los problemas de comunicación, termina por ocasionarle 
situaciones de incomodidad, aislamiento e, incluso, problemas de autoestima que van a repercutir negativamente en futuras relaciones de tipo social con el mundo oyente. De ahí que Keilmann, Limberger, y Mann (2007) añadan que las personas con sordera sean más propensos a una baja autoestima, menos capacidad de socialización y se sientan menos aceptados. No resulta extraño, por ende, que su desarrollo socioemocional sea inapropiado o limitado (Bandura, 1977).

Pero, en este punto cabe destacar, que al igual que no se puede enseñar a leer a una persona, si uno mismo/a no está alfabetizado y/o no conoce las estrategias educativas necesarias para hacerlo, esto tampoco resultará posible en el ámbito de la alfabetización emocional (Valsameda, 2009).

\section{Método}

Partiendo de premisas anteriormente expuestas, en el presente estudio se pretende llevar a cabo una revisión sistemática de las investigaciones sobre competencia emocional (identificación, expresión, comprensión de emociones, regulación de sentimientos) de escolares con sordera publicadas en los últimos 10 años en revistas indexadas en las bases de datos Scopus, PubMed y Dialnet.

Para el proceso de la misma, se introdujeron las palabras clave relacionadas con el mundo de las emociones y la discapacidad auditiva, como: emotion, emotional education, deafness, deaf children, emotional alphabetization, emotional intelligence, emocional programs, deaf emotion, deaf students y hearing impairment.

La primera estrategia de búsqueda fue filtrar la información por año, es decir, en las bases de datos mencionadas se acotó la búsqueda a los últimos 5 años, aunque este filtro se tuvo que ampliar a 10 años por las insuficientes investigaciones con el tema tratado.

En PubMed se comenzó la búsqueda con las palabras clave emotional education AND deaf children, y como no se encontró ningún artículo se cambiaron los términos deaf children por deafness y/o hearing impairment, emotional education por emotional alphabetization, continuando con emotional intelligence AND deafness y/o hearing impairment, y se encontraron un total de 8 artículos. Posteriormente, centrando la búsqueda con los términos deaf children AND emotional intelligence y/o emotional programs, los artículos se redujeron a la mitad.

Después, con emotion AND deafness se obtuvieron un total de 37 artículos, pero se redujeron a 14 cuando se cambió la palabra clave deafness por deaf children y/o deaf students. Y, finalmente, con las palabras clave deaf emotion AND deaf children se consiguieron 23 artículos.

Aparte de lo indicado, en esta primera fase y una vez recopilados los artículos por medio de las palabras clave anteriormente mencionadas, se tuvieron en cuenta el título, el resumen y los resultados de los estudios.

En la base de datos Scopus, la búsqueda se realizó con los mismos términos y la misma secuencia que la anterior, aunque en este caso con las palabras clave emotional intelligence AND deafness y/o hearing impairment, se localizaron 10 artículos, pero al centrar la búsqueda a deaf children los resultados disminuyeron a 4 . Se prosiguió con deaf children AND emotional programs, pero no se localizó ningún artículo. 
Luego, con los términos emotion AND deafness se encontraron un total de 104 artículos, pero al cambiar la palabra clave deafness por deaf children los mismos se redujeron a 38 .

Y de igual forma que el anterior, y en una primera fase, se tuvieron en cuenta el título, el resumen y los resultados de los estudios.

En la base de datos Dialnet, se utilizaron las mismas palabras clave, aunque escritas en castellano. Se empezó así, con educación emocional y discapacidad auditiva y/o sordera, educación emocional y niños sordos, déficit auditivo y alfabetización emocional. Luego, se optó por programas sobre la educación emocional y/o inteligencia emocional y sordera, o programas sobre la educación emocional y/o inteligencia emocional y niños sordos.

Después a través de las palabras clave inteligencia emocional y niños sordos, se localizó 1 artículo. Y con emoción y niños sordos se obtuvieron un total de 3 , de los cuales uno de ellos era el mismo que ya se había localizado anteriormente.

Los criterios de inclusión empleados para la selección de los artículos fueron: El criterio del tema: documentos relacionados con la competencia emocional en escolares sordos.

El criterio temporal: artículos publicados en los últimos 5 años, aunque ese margen se tuvo que ampliar a 10 años debido al escaso número de artículos encontrados.

El criterio de acceso: documentos completos y disponibles por medio del catálogo de la biblioteca de la UOC, Universidad de Vigo y biblioteca pública central de Juan Companel.
El criterio del idioma: estudios escritos en inglés, italiano, portugués $\mathrm{O}$ castellano.

Se eliminaron artículos de acuerdo a los siguientes criterios de exclusión:

Artículos con muestras de sujetos en edad no escolar.

Investigaciones que incluían escolares con síndromes que cursan con sordera (Pendred, Hurler...).

Artículos irrelevantes, repetidos, y no disponibles a texto completo en aquellas fuentes citadas en el criterio acceso.

El análisis de los artículos se llevó a cabo utilizando los siguientes parámetros: autor y año, participantes, propósito, evaluación, diseño metodológico, resultados y limitaciones.

Con referencia a los participantes, se clasificaron en dos o tres grupos en función de si eran estudiantes sordos, oyentes o tenían IC. Se añadió, además, el país donde se llevaron a cabo los diferentes estudios. Con respecto al propósito, se puso el objeto de estudio de cada artículo. Con la evaluación, se describieron los instrumentos y el tipo de medidas utilizadas. En el diseño metodológico, se explicitó si se trataba de estudios comparativos, intervenciones, etc. En los resultados, se analizaron los descritos de cada estudio en base a la competencia emocional que analiza cada uno. Por otro lado, en las limitaciones, fueron señaladas las barreras que encontraron los investigadores en sus estudios, y para finalizar, el apartado de conclusiones donde se desarrollará una comparativa sobre los estudios analizados junto con aportaciones de autores del ámbito científico sobre la temática que resulten relevantes. 
Tabla 1. Artículos encontrados y seleccionados

\begin{tabular}{|c|c|c|c|}
\hline \multicolumn{4}{|c|}{ Patrón de Busqueda } \\
\hline IDENTIFICADOS & $\begin{array}{l}\text { PUBMED } \\
\text { DOCUMENTOS } \\
\text { IDENTIFICADOS } \\
(\mathbf{n = 6 9 )} \\
\end{array}$ & $\begin{array}{l}\text { SCOPUS } \\
\text { DOCUMENTOS } \\
\text { IDENTIFICADOS } \\
(\mathbf{n = 1 1 4})\end{array}$ & $\begin{array}{l}\text { DIALNET } \\
\text { DOCUMENTOS } \\
\text { IDENTIFICADOS } \\
(\mathbf{n = 3})\end{array}$ \\
\hline EXCLUÍDOS & $\begin{array}{l}\text { Documentos } \\
\text { excluidos } \\
\text { Irrelevantes ( } \mathrm{n}=53) \\
\text { Muestras sólo } \\
\text { sujetos i.coclear } \\
(\mathrm{n}=10) \\
\text { Déficits asociados: } \\
(\mathrm{n}=1)\end{array}$ & $\begin{array}{l}\text { Documentos excluidos } \\
\text { Irrelevantes }(\mathrm{n}=85) \\
\text { Muestras solo sujetos con } \\
\text { i.coclear }(\mathrm{n}=10) \\
\text { Déficits asociados }(\mathrm{n}=2) \\
\text { No disponibles a texto } \\
\text { completo }(\mathrm{n}=3) \quad \begin{array}{l}\text { No en edad escolar }(\mathrm{n}=6) \\
\text { Repetidos }(\mathrm{n}=4)\end{array}\end{array}$ & $\begin{array}{l}\text { Documentos } \\
\text { excluidos } \\
\text { Irrelevantes }(n=1) \\
\text { No disponibles a } \\
\text { texto completo }(n= \\
\text { 1) } \\
\text { Repetidos }(n=1)\end{array}$ \\
\hline INCLUÍDOS & $\begin{array}{l}\text { PUBMED } \\
\text { DOCUMENTOS } \\
\text { INCLUIDOS } \\
(\mathrm{n}=6)\end{array}$ & $\begin{array}{l}\text { SCOPUS } \\
\text { DOCUMENTOS } \\
\text { INCLUIDOS } \\
(\mathrm{n}=4)\end{array}$ & \\
\hline & $\begin{array}{l}\text { DOCUMENTOS I } \\
\text { BASES DE DATO } \\
\text { DOCUMENTOS F } \\
\text { INCLUIDOS }(\mathbf{n}=\mathbf{1} \\
\end{array}$ & $\begin{array}{l}\text { CLUIDOS DE TODAS LAS } \\
(\mathrm{n}=10) \\
\text { NALMENTE }\end{array}$ & \\
\hline
\end{tabular}

\section{Resultados}

Finalizada la revisión sistemática sobre el desarrollo emocional de escolares con sordera entre los años 2006 y 2016 fueron seleccionados y analizados diez artículos.

A través del proceso de revisión se constata que se han llevado a cabo investigaciones en varios continentes. De los diez estudios, cinco se llevaron a cabo en Europa, tres en Asia y dos en América.

Con respecto a los participantes, las muestras varían entre 10 y 426 sujetos, registrándose la muestra más pequeña en el estudio de Lori (2014) y la más grande en el de Hintermair (2007). (Ver Apéndice).

Atendiendo al propósito, el $50 \%$ de los artículos analizó cómo identifican, atribuyen, reconocen y regulan las emociones básicas (felicidad, tristeza, miedo y cólera) los escolares con sordera (con o sin IC) en comparación a cómo lo hacen los oyentes, estudiando aspectos relacionados con la comprensión, regulación e identificación de emociones (Rieffe, 2012; Wiefferink 2012), la atribución de las mismas en los diferentes contextos (Gray et al., 2007; Rieffe, 2012), y sumándole a este último la identificación emocional (Ziv, Most, y Cohen, 2013). Estas investigaciones hacen mención a la comprensión de emociones (Ziv et al., 2013), a la relación entre la capacidad de reconocimiento facial de emociones de niños sordos con las habilidades lingüísticas, las características de la sordera y el nivel educativo de los padres (Sidera, et al., 2016) y dos de ellos a la regulación emocional (Rieffe, 2012; y Ziv et al., 2013). Resulta relevante señalar que la diferencia entre identificación y comprensión de 
emociones en estos artículos reside en la consideración de esta última como la habilidad para entender el lenguaje emocional y el conocimiento acerca de las emociones, relacionando esto con la inferencia de las emociones en contextos sociales, el cual requiere un razonamiento previo, resultando un proceso más complejo que la mera identificación.

El $20 \%$ de los estudios investigaron el desarrollo social y emocional (Lori, 2014; Vogel-Walcutt, Schatschneider, y Bowers, 2011) y el 10\% el diagnóstico y prevalencia de problemas socioemocionales, de niños y niñas sordas (Hintermair, 2007) y el 20\% restante las diferencias en inteligencia emocional entre el alumnado sordo y oyente (Hanafi, Yasin, Bari, y Salubin, 2012; Pourmohamadreza-Tajrishi, Ashori, y Jalilabkenar, 2013).

Respecto al diseño metodológico seguido en nueve de los diez estudios, se basó bien en una comparación entre escolares oyentes y sordos (con o sin IC), mientras que en el estudio restante se llevó a cabo una intervención únicamente con estos últimos de una edad comprendida entre los 12 y 14 años de edad.

Los resultados se agruparon según los distintos aspectos de la competencia emocional: identificación, comprensión y atribución de emociones, regulación emocional, inteligencia emocional $\mathrm{y}$ desarrollo socioemocional de los escolares sordos.

Con respecto a la identificación de las emociones, los resultados de uno de los tres estudios que hacen referencia a este proceso, muestran tanto en sujetos sordos como en oyentes mayores dificultades para reconocer (miedo, sorpresa y asco) que (felicidad, tristeza $\mathrm{y}$ enfado) en ambos grupos, compartiendo el mismo orden de dificultad en el reconocimiento de las mismas. Asimismo se refleja que el grupo de sujetos sordos se retrasó en variables lingüísticas del vocabulario y las habilidades lingüísticocomunicativas, correlacionando estas variables significativamente con su capacidad para reconocer las emociones. Por ende, las habilidades lingüísticas se relacionan con las habilidades de reconocimiento de emoción (Sidera et al, 2016; Wiefferink (2012).

En cambio, los dos estudios restantes desvelaron que los escolares sordos (con o sin IC) reconocen menos emociones que los oyentes, presentando un nivel intermedio de dificultad para reconocer el miedo y la cólera y una mayor dificultad para la sorpresa y el asco (Rieffe, 2012; Wiefferink et al., 2012; y Ziv et al., 2013). Si bien, las emociones más reconocidas tanto por sujetos oyentes como por sordos (con o sin IC) fueron la felicidad seguida de la tristeza (Rieffe, 2012; Wiefferink et al., 2012; y Ziv et al. 2013), y las más difíciles: la sorpresa, el miedo, el asco y el enfado (Ziv et al. 2013).

Del mismo modo que el alumnado oyente, los niños con DA mostró mayor dificultad a la hora de distinguir emociones dentro del dominio negativo (tristeza y cólera, por ejemplo) que a través de una valencia contraria (felicidad y tristeza) (Rieffe, 2012; Wiefferink et al., 2012).

En cuanto a la atribución de emociones a diferentes contextos, la tasa de éxito en los niños oyentes fue significativamente mayor que en aquellos con sordera, desacelerando esta tasa a medida que aumenta la edad de este último grupo, interpretando el retraso en el desarrollo en la comprensión emocional de los niños 
con DA en términos de la experiencia social de los niños sordos de padres oyentes, lo cual les permite una capacidad limitada para analizar la causalidad emocional (Gray et al., 2007).

En referencia a la comprensión emocional (identificación de las caras, comprensión de las emociones en contextos típicos y perspectiva afectiva de historias, se muestra que la mayoría de los alumnos y alumnas oyentes obtuvieron mejores resultados a la hora de comprender las emociones que el grupo del alumnado sordo con o sin IC (Ziv et al., 2013). Por otra parte, el retraso en la comprensión de las emociones en los niños sordos con o sin implante podría explicarse por la menor calidad y cantidad de la interacción entre los padres y sus hijos y por la exposición más baja y menos modelada de los adultos antes de que se produjera la implantación coclear (Wiefferink et al. 2012).

En cuanto a la regulación de las emociones, los resultados de los estudios de Rieffe (2012) y Wiefferink et al. (2012), demostraron que el grupo del alumnado con sordera tiene las habilidades de empatía (la habilidad para estar consciente de, reconocer, comprender y apreciar los sentimientos de los demás) en un nivel inferior que los oyentes, señalando que las habilidades del lenguaje guardan relación con la competencia social o conductas empáticas en niños con IC. Asimismo Wiefferink (2012) establece una relación en el alumnado oyente entre la regulación emocional y el funcionamiento social, cosa que no ocurre en el alumnado con IC de su estudio.

Con respecto al desarrollo socioemocional de los/as sujetos con sordera (con o sin IC), los resultados de dos de los diez estudios demostraron, por un lado, que estos estudiantes tienen un desarrollo emocional pobre, y mayor frecuencia de problemas emocionales que los estudiantes oyentes (Hintermair, 2007), apuntando que pese a desarrollar una intervención relativamente temprana, el uso apropiado de los dispositivos y la aplicación de un sistema oral-auditivo sigue habiendo un posible riesgo de problemas de desarrollo socio-emocional a temprana edad en algunos niños con pérdida auditiva neurosensorial permanente (Lori, 2014), atribuible según Hintermair (2007) a la relevancia de los recursos parentales para la cantidad de estrés experimentado por los padres como un factor determinante para el desarrollo socio-emocional de sus hijos, señalando además que muchas escuelas carecen de personal que esté suficientemente formado en psicoterapia.

Sin embargo, por otra parte los resultados del estudio de Vogel-Walcutt et al. (2011) no obtuvieron diferencias significativas entre ambos grupos, justificando que el alumnado con sordera cursa con otros retrasos significativos en el desarrollo, por lo que no se pueden dar razones claras para estas diferencias, aludiendo a los posibles errores de muestreo que se dan en este tipo de estudios (diferencias en metodología, edad, idioma o tipo de escuela).

Por otro lado, los resultados de los dos artículos que investigaron la inteligencia emocional, revelaron que los sujetos con sordera tienen habilidades de inteligencia emocional bajas (Hanafi et al, 2012), y que la IE tiene efectos positivos para la salud mental de dichos sujetos, reduciéndose, por tanto, situaciones de ansiedad y depresión (Pourmohamadreza-Tajrishi et al., 2013). 
Finalmente, las limitaciones descritas por los autores de los artículos fueron: la necesidad de desarrollar estudios longitudinales que exploren a los niños con IC, dado que la mayoría han sido implantados recientemente por lo que existe un menor número de experiencias auditivas en relación a los sujetos oyentes (Wiefferink et al., 2012), una baja tasa de respuesta en los cuestionarios empleados (Hintermair, 2007; Vogel et al., 2011),la utilización de muestras pequeñas (Hintermair, 2007; Lori, 2014; Vogel et al., 2011; Rieffe, 2012; y PourmohamadrezaTajrishi et al., 2013) y en dos de los estudios encontraron ínfimo el número de emociones analizadas, considerando que atenerse a un número tan limitado acota los resultados (Gray et al., 2007; Wiefferink et al., 2012). Esto restringe la posibilidad de generalizar los datos. Asimismo, se hace referencia al empleo de tareas que implican únicamente el reconocimiento facial de caras estáticas y no humanas. (Sidera, et al, 2016).

La distribución los artículos según los parámetros anteriormente citados, puede observarse en la Tabla A1, desarrollada en el apéndice.

\section{Discusión y conclusions}

Los estudios revisados refrendan que los escolares con sordera (con o sin IC) tienen un desarrollo emocional y unas habilidades socioemocionales inferiores si se comparan con las del alumnado oyente en aquellos aspectos desarrollados con anterioridad (regulación, comprensión, atribución de emociones). Si se cotejan muestras de discentes con sordera con aquellas de sujetos oyentes, se encuentra que las primeros presentan una habilidad elemental o limitada a la hora de identificar, comprender y regular las emociones básicas debido a la falta de entrada verbal a edades tempranas
(Cañizares, 2015) y por una calidad empobrecida de interacciones con la gente oyente en su entorno inmediato (Farrugia, 1982; Peterson y Siegal, 2000; Quirin y Lane, 2012). Estos aspectos han sido corroborados por otras investigaciones que han demostrado que los niños con pérdida de la audición, incluidos aquellos que padecen pérdidas unilaterales, pueden estar en riesgo de encontrar problemas socio-emocionales (Dammeyer, 2010, Hogan, Shipley, Strazdins, Purcell y Baker, 2011; Tharpe, 2008). En este colectivo algunos investigadores incluyen aquellos casos en los que se desarrolla una identificación temprana pero una intervención educativa insuficiente que no consiga paliar los retrasos en el desarrollo del habla y el lenguaje (Ching et al.,2013), los cuales repercuten por ejemplo, como se ha referenciado anteriormente en la relevante relación que guardan las habilidades lingüísticas con las competencias de reconocimiento de emociones (Sidera et al, 2016).

Por otra parte, en un estudio longitudinal, los autores YoshinagaItano, Baca y Sedey (2010) afirman que la capacidad de lenguaje receptivo en niños con pérdida auditiva de grave a profunda con o sin IC resulta similar en comparación con los datos normativos sobre los niños con audición, presentando únicamente siete meses de retraso en el vocabulario expresivo, lo cual induce a concluir que la calidad de la intervención médica y educativa resulta un factor determinante en la consecución de un óptimo desarrollo de las habilidades comunicativas y socioemocionales, dado que muchos niños con IC son capaces de desarrollar habilidades de lenguaje proporcionables con compañeros con audición normal (Svirsky, et al., 2000), dejando atrás problemas de atención dividida, una adquisición más tardía del lenguaje, la 
suplencia mental a causa de una lectura labio facial que puede causar confusiones en determinados fonemas, entre otros (Lledo, 2008). A mayores de la disposición médica y educativa mencionada, la sensibilidad y la capacidad de respuesta de los padres durante los primeros años de vida, contribuirá como uno de los requisitos previos esenciales en el proceso de desarrollo de los infantes (Wiefferink, 2012), así como la utilización de un sistema de comunicación apropiado, funcional y centrado en el niño, sin que importe la modalidad del mismo (Dammeyer, 2010.)

De este modo, los datos de la presente revisión han demostrado que la falta de audición dificulta la comprensión de las emociones ya que, generalmente, los niños/as con sordera están expuestos/as a un ínfimo número de explicaciones sobre el porqué de las cosas que se desarrollan a su alrededor y de profundizaciones acerca de las experiencias emocionales que vivencian y los sentimientos que estas les generan (Muñoz, 2006).

Las dificultades emocionales de niños y niñas con DA como constituye por ejemplo, una inferior habilidad empática (Rieffe ,2012); Wiefferink et al. ,2012), les dificulta ponerse en el lugar del otro, generándoles en ocasiones conflictos en las relaciones interpersonales (Keilmann, Limberger, y Mann, 2007; Quirin y Lane, 2012).

Por todo lo dicho, y coincidiendo con Valmaseda (2009), se considera necesario fomentar en el alumnado con sordera dichas destrezas emocionales para que ellos/as mismos sean capaces de discriminar acertadamente las emociones expresadas por los demás, etiquetar las mismas y reconocer en qué categorías se agrupan los sentimientos, así como regular las emociones propias y ajenas, moderando las sensaciones negativas e intensificando las positivas, ya que si son capaces de realizar todo ello, posibilitarán un crecimiento y desarrollo armónico en lo emocional e intelectual.

Las dificultades emocionales de los niños con dificultades auditivas puede ser mejoradas por programas educativos de IE (Salovey, 2007), dado que misma al igual que la capacidad o habilidad cognitiva puede entrenarse a través de tareas que impliquen el procesamiento de la información emocional, evitando problemas psicológicos en dicha población, ayudándoles de este modo a regular y controlar sus emociones (Bisquerra, 2003). A modo de ejemplo, hacer referencia al programa de alfabetización emocional "Desconócete a ti mismo" de Güell y Muñoz (2000).

Adicionalmente, esta revisión apunta que el desarrollo de los estudios que abordan esta temática en diferentes países supone que la materia de las emociones en escolares con discapacidad auditiva ha sido explorada en diferentes zonas del mundo, lo cual resulta positivo dado que induce a la idea de que despierta el interés y la preocupación en diversas culturas.

Y si bien, las investigaciones realizadas presentan algunos hallazgos que favorecen el conocimiento a cerca de la competencia emocional que experimentan las personas con discapacidad auditiva, se hace necesario ahondar en la investigación de esta temática, posibilitando una visión más profunda y compleja de la misma, mitigando las limitaciones encontradas en los estudios analizados (muestras pequeñas, una insuficiente variedad de tipologías emocionales, estudios no longitudinales, diferencias metodológicas, de edad, idioma o tipo de escuela,...). Por ende, estas mismas restricciones repercuten en la presente 
revisión planteada, puesto que mientras unos datos resultan unánimes por varios autores y apoyados por investigaciones o teorías científicas anteriores, otros son tachados por los propios investigadores como carentes de haber obtenido un suficiente rigor científico y por tanto, datos generalizables, como ocurre en el caso de Vogel-Walcutt et al. (2011).

De hecho, como se ha comentado con anterioridad, hasta el momento la cultura occidental, ha confiado más en la razón que en cualquier otra dimensión de la persona. Se ha educado al alumnado sordo poniendo el foco de atención en aspectos lingüísticos o culturales, dejando al margen el repertorio emocional que lo conforma. Esto es, se le han enseñado y priorizado los razonamientos lógicos, dejando el margen de los mismos el desarrollo de una buena competencia emocional. Sin embargo, las nuevas tendencias en educación y los estudios sobre la inteligencia emocional, han puesto en evidencia que las habilidades que conducen a una vida plena están fundamentalmente condicionadas por aspectos de tipo emocional. De este modo, lo que sienten las personas con sordera sobre sus experiencias de aprendizaje, debe ser tan importante como lo que aprenden dentro de las instituciones educativas, porque es probable que, si las estrategias de regulación sobre los sentimientos que originan nuestras emociones no se desarrollan bien en los primeros años de vida, los niños/as sordos adquirirán un nivel de gestión subóptimo (Goleman, 2001).

Como se ha visto, los escolares con sordera, aunque varíen extensamente en personalidad, intereses y salud mental, pueden presentar dificultades en su desarrollo socio-emocional (Bandura, 1977) debido a los problemas relacionados con la comunicación
(Quirin y Lane, 2012) lo cual influye de manera negativa en su salud mental (Bar-on, 2006) y los sitúa en una posición de desventaja, con respecto a la población oyente.

Como consecuencia de todo ello, las personas con discapacidad auditiva pueden mostrarse más inseguras, egocéntricas, impulsivas, con un pobre autoconcepto, inmaduras socialmente y poco empáticas. Características, todas ellas, de un desarrollo personal inmaduro producto de las interacciones con el medio social en el que crecen. Es decir, el retraso en su desarrollo emocional no se deriva de la sordera en sí misma, sino de formar parte de un ambiente que no se adapta a las necesidades lingüísticas que demanda esta población (Simón, 2007).

En este sentido, las implicaciones educativas deben partir de un estado de concienciación, haciendo más sensibles a familias y escuela sobre la importancia de desarrollar la competencia emocional y el peso que ésta tiene en la formación y desarrollo armónico de los alumnos y alumnas que padecen sordera.

La escuela, en este aspecto, debe optar por un modelo educativo donde haya cabida para el desarrollo de este ámbito, ya que si desde las aulas se trabaja la dimensión social y afectiva de este alumnado, su desarrollo integral mejorará sustancialmente.

Como apunta Pineda (2010, p. 176) "las personas, al igual que las aves, son diferentes en su vuelo, pero iguales en su derecho a volar".

\section{Referencias}

Bandura, A. (1977). Social learning theory. NJ: Prentice Hall. 
Bar-on, R. (2006). The Bar-on model of emotional-social intelligence (ESI). Psicothema, 18(1), 13-25.

Benvenuto, A. (2009). El sordo y lo Inaudito. Uruguay: Educa. Recuperado el 16 de Noviembre del 2016, de:http://www.uruguayeduca.edu.uy/ Userfiles/P0001/File/El\%20sordo\%2 0y\%201o\%2 0inautito.doc

Boix, C. (2007). Educar para ser feliz. Una propuesta de Educación Emocional. Madrid. CEPE.

Cañizares, G. (2015). Alumnos con déficit auditivo. Un nuevo método de enseñanza- aprendizaje. Madrid. Narcea, S.A. de Ediciones.

Dammeyer, J. (2010). Psychosocial development in a Danish population of children with cochlear implants and deaf and hard-of-hearing children. Journal of Deaf Studies and Deaf Education, 15(1), 50-58.

Denham, S. A., Blair, K. A., DeMulder, E. D.,

Levitas, J., Sawyer, K., AuerbachMajor, S., Queenan, P. (2003). Preschool emotional competence: Pathway to social competence? Child Development, 74 (1), 238-256.

Díaz, E. (2013). El reflejo de la mujer en el espejo de la discapacidad. La conquista de los derechos sexuales y reproductivos de las mujeres con discapacidad. Madrid: Cinca Editorial. Recuperado el 2 de Noviembre del 2016, de: http://www.once.es/new/plan-deigualdad/documentos-de interes/El\%20reflejo\%20de\%20la\%2 0mujer\%20en\%20el\%20espejo.pdf
Farrugia, D., y Austin, G. (1982). A study of social-emotional adjustment patterns of hearing impaired students in different educational settings. American Annals of the Deaf 125, 535-41.

Fehr, B. J. y Exline, R. V. (1987). Social visual interactions: A conceptual and literature review. En A. W. Siegman \& S. Feldstein (Eds.), Nonverbal behaviour and communication (Vol. 2nd, pp. 225326). Hillsdale, NJ: Lawrence Erlbaum.

Flavell, J.H. (2004). Theory-of-Mind Development: Retrospect and Prospect. Merrill-Palmer Quarterly, July 2004, Vol. 50, No. 3, 274-290. 1.

Goleman, D. (2001) Inteligencia Emocional. Barcelona. Kairós. Gray, C., Hosie, J., Russell, P., Scott, C. Hunter, N. (2007). Attribution of emotions to story characters by severely and profoundly deaf children. Journal of Developmental and Physical Disabilities, 19 (2), 145-159.

Güell, M. Muñoz, J. (2000). Desconócete a ti mismo. Programa de alfabetización emocional. Barcelona. Paidos

Gutiérrez Fernández, A. (2007) El futuro de la educación del sordo. Y mientras, algunos retos, necesidades y demandas del presente. Psicología Educativa Vol. 13 (1) pp. 5-34. Recuperado el 5 de Noviembre del 2016, de: http://docplayer.es/22008086Psicologia-educativa-2007-vol-13-no-1-pags-5-34-issn-1135-755.html

Hanafi, M., Yasin, M., Bari, S., Salubin, R. (2012). Emotional intelligence 
among deaf and hard of hearing children. Social Sciences, 7 (5), pp. 679-682.

Hintermair, M. (2007). Prevalence of socioemotional problems in deaf of hearing children in Germany. American Annals of the Deaf, 152 (3), 320-330.

Hogan, A., Shipley, M., Strazdins, L., Purcell, A., \& Baker, E. (2011). Communication and behavioural disorders among children with hearing loss increases risk of mental health disorders. [Comparative Study]. Australian and New Zealand Journal of Public Health, 35(4), 377383.

Keilmann, A., Limberger, A., y Mann, W.J. (2007). Psychological and physical well-being in hearingimpaired children. International Journal of Pediatric Otorhinolaryngology, $71 \quad$ (11), pp.1747-1752.

Keltner, D. y Gross, J. (1999) Functional account of emotions. Cognition and Emotion Special Issue: Functional Account Of Emotion. 13(5) 467-480.

Ketelaar, L., Wiefferink, C.H., Frijins, J.H.M., Broekhof, E., Rieffe, C. (2015). Preliminary findings on associations between moral emotions and social behavior in young children with normal.

Quirin, M., \& Lane, R. D. (2012). The construction of emotional experience requires the integration of implicit and explicit emotional processes. Behavioral and Brain Sciences, 35, 159-160.hearing and with cochlear implants. European Child and Adolescent Psychiatry, 24 (11), pp. 1369- 1380.
Li, Y., Bain, L., \& Steinberg, A. G. (2003). Parental decision making and the choice of communication modality for the child who is deaf. [Comparative Study Research Support, Non-U.S. Gov't]. Archives of Pediatrics and Adolescent Medicine, 157(2), 160-168.

Lledo, A. (2008). La discapacidad auditiva. Un modelo de educación inclusiva. Madrid. Edebe

Lori, H. (2014). Social-Emotional Development in Children with Hearing Loss. Theses and Dissertations-Communication Sciences and Disorders. Paper 4. Recuperado el 2 de Enero del 2017 de:

http://uknowledge.uky.edu/commdis orders_etds/4

Molina, L., Ipiña, M., Reyna, C. y Guzmán, R. (2011). Competencia social en niños con sordera profunda. Revista CES Psicología, 4, 1-14.

Muñoz, J. (2006). Sordera y salud mental: la psicología frente a la deficiencia auditiva. Infocop On/ine.

Pabón, S. (2009). La discapacidad auditiva. ¿Cómo es el niño sordo?. Revista internacional de innovación y experiencias educativas, 16 (305), $1-10$.

Peterson, C. y Siegal, M. (2000). Insights into Theory of Mind from Deafness and Autism. Mind \& Language, 15: 123-145.

Pigliacampo, R. (2007). Parole nel movimento. Psicolinguistica del sordo. Italia. Armando Editore.

Pineda, P. (2015). Niños con capacidades especiales. Madrid. Hércules Ediciones. 
Pourmohamadreza, M., Ashori, M., \& Jalilabkenar, S. (2013). The Effectiveness of emotional intelligence training on the mental health of male deaf students. Iranian journal of public health, 42(10), 1174.

Ramos, N., Recondo, O. y Henríquez, H. (2012) Practica la Inteligencia Emocional Plena: la gestión eficaz de las emociones a través de mindfulness. Barcelona. Kairós.

Rieffe, C. (2012). Awareness and regulation of emotions in deaf children. British Journal of Developmental Psychology, 30(4), 477-492.

Rieffe, C., Terwogt, M., and Jellesma, F. (2008). Emotional competence and health in children. Emotion regulation, 184-201.

Riso, W. (2012). Sabiduría emocional. Un reencuentro con las fuentes naturales del bienestar y la salud emocional. Nápoles. Océano.

Schutte, N.S., Malouff, J.M., Thorsteinsson, E.B., Bhullar, N., Rooke, S.E. (2007). Personality and individual differences, 42 (6), 921933.

Sidera, F., Amadó, A. y Martínez, L. (2016) Influences on Facial Emotion Recognition in Deaf Children. Journal of Deaf Studies and Deaf Education, 1-14.

Simón, M. (2007). Educación y desarrollo socioemocional en los niños sordos. Ríos. Educa

Svirsky, M. A., Robbins, A. M., Kirk, K. I., Pisoni, D. B., \& Miyamoto, R. T. (2000). Language development in profoundly deaf children with cochlear implants. [Research Support, U.S. Gov't, P.H.S.]. Psychological Science, 11(2), 153158.

Tharpe, A. M. (2008). Unilateral and mild bilateral hearing loss in children: Past and current perspectives. [Historical Article Research Support, Non-U.S. Gov't Research Support, U.S. Gov't, NonP.H.S. Review]. Trends Amplif, 12(1), 7-15.

Valmaseda, M. (2004). El desarrollo socio-emocional de los niños sordos. Intervención desde la escuela. En A.B. Domínguez y P. Alonso (Eds.) La educación de los alumnos sordos hoy. Perspectivas y respuestas educativas. Málaga: Aljibe.

Valmaseda, M. y Gómez, L. (1999). La intervención educativa con los alumnos sordos. En J.N. García Sánchez (Coord.). Intervención psicopedagógica en los trastornos del desarrollo. Madrid: Pirámide

Vogel-Walcutt, J.J., Schatschneider, C., Bowers, C. (2011). Social-Emotional Functioning of Elementary-Age Deaf Children: A Profile Analysis. American Annals of the Deaf, 156 (1), 6-22.

Wiefferink, C.H., Rieffe, C., Ketelaar, L., Frijins, J.H. (2012). Predicting social functioning in children with a cochlear implant and in normalhearing children: The role of emotion regulation. Internacional Journal of Pediatric Otorhinolaryngology, 76 (6), 883-889.

Yoshinaga-Itano, C., Baca, R. L., \& Sedey, A. L. (2010). Describing the trajectory of language development in the presence of severe-to-profound 
hearing loss: A closer look at children with cochlear implants versus hearing aids. Otology \& Neurotology, 31(8), 1268-1274.

Ziv, M., Most. T., Cohen, S. (2013). Understanding of emotions and false beliefs among hearing children versus deaf children. Journal of Deaf Studies and Deaf Education, 18 (2), 161-174. 


\begin{tabular}{|c|c|c|c|c|c|c|}
\hline \multicolumn{7}{|c|}{$\begin{array}{l}\text { Apéndice } \\
\text { TABLA A1. Estudios incluidos en esta revisión }(n=10) / / \text { Análisis de los estudios seleccionados. }\end{array}$} \\
\hline POBLACIÓN & $\begin{array}{c}\text { AUTORES Y } \\
\text { AÑO DE } \\
\text { PUBLICACIÓN } \\
\end{array}$ & PROPÓSITO & EVALUACIÓN & $\begin{array}{l}\text { DISEÑO } \\
\text { METODOLÓGICO }\end{array}$ & RESULTADOS & LIMITACIONES \\
\hline $\begin{array}{l}\text { España } \\
\mathrm{N}^{\mathrm{0}}= \\
\text { participantes }(75) \\
\text { sordos de edad 3- } \\
8 \text { años. }\end{array}$ & $\begin{array}{l}\text { Sidera, F., Amadó, } \\
\text { A. y Martínez, L. } \\
(2016) .\end{array}$ & $\begin{array}{lr}\text { Investigar } & \text { la } \\
\text { relación entre la } & \text { lacidad de } \\
\text { capacido } & \\
\text { reconocimiento } \\
\text { gestual de } \\
\text { emociones de } \\
\text { niños sordos con } \\
\text { las habilidades } \\
\text { lingüísticas, las } \\
\text { características de } \\
\text { la sordera y el } \\
\text { nivel educativo de } \\
\text { los padres. }\end{array}$ & $\begin{array}{l}\text { Pruebas: } \\
\text { Mann-Whitney's } \\
\text { Spearman } \\
\text { Kruskal-Wallis } \\
\text { Chi-square }\end{array}$ & $\begin{array}{l}\text { Diseño comparativo } \\
\text { entre niños sordos de } \\
\text { diferentes edades y } \\
\text { niños oyentes. }\end{array}$ & $\begin{array}{l}\text { Se relacionó el nivel educativo } \\
\text { de las madres con las } \\
\text { habilidades de reconocimiento } \\
\text { de emoción. } \\
\text { El grupo de sordos se retrasó en } \\
\text { variables lingüísticas del } \\
\text { vocabulario y las habilidades } \\
\text { lingüístico-comunicativas, y } \\
\text { estas variables } \\
\text { correlacionaron } \\
\text { significativamente con su } \\
\text { capacidad para reconocer las } \\
\text { emociones. } \\
\text { Mayores dificultades para } \\
\text { reconocer (miedo, sorpresa y } \\
\text { asco) que (felicidad, tristeza y } \\
\text { enfado) en ambos grupos. } \\
\text { Las habilidades lingüísticas se } \\
\text { relacionan con las habilidades } \\
\text { de reconocimiento de emoción. } \\
\text { Niños sordos y oyentes } \\
\text { comparten el mismo orden de } \\
\text { reconocimiento de emociones. }\end{array}$ & $\begin{array}{l}\text { Las tareas } \\
\text { implicaron } \\
\text { únicamente } \\
\text { reconocimiento } \\
\text { verbal de caras. } \\
\text { Empleo de dibujos } \\
\text { de expresiones } \\
\text { faciales que no } \\
\text { parten de contextos } \\
\text { significativos para el } \\
\text { niño (por ejemplo, } \\
\text { una foto de la cara } \\
\text { de su madre } \\
\text { enfadada). }\end{array}$ \\
\hline $\begin{array}{l}\text { Estados Unidos } \\
\text { Dos niñas y tres } \\
\text { niños con sus } \\
\text { respectivos } \\
\text { padres/madres. }\end{array}$ & Lori, H. (2014). & $\begin{array}{l}\text { Describir la } \\
\text { capacidad social y } \\
\text { emocional en } \\
\text { niños (edades de } \\
3.0-6.6 \text { años) con } \\
\text { pérdida auditiva } \\
\text { neurosensorial } \\
\text { que utilizan }\end{array}$ & $\begin{array}{l}\text { Pipps } \\
\text { (instrumento de } \\
\text { calificación de } \\
\text { comportamiento) } \\
\text { Likert-scale } \\
S C B E \text { (escala de } \\
\text { observación) } \\
B A S C-2\end{array}$ & $\begin{array}{l}\text { Diseño comparativo } \\
\text { entre niños con } \\
\text { sordera de diferentes } \\
\text { edades y niños con } \\
\text { audífonos y/o IC. }\end{array}$ & $\begin{array}{l}\text { A pesar de } \\
\text { intervención relativamente } \\
\text { temprana, el uso apropiado de } \\
\text { los dispositivos y la aplicación } \\
\text { de un sistema oral-auditivo } \\
\text { sigue habiendo un posible riesgo } \\
\text { de problemas de desarrollo } \\
\text { socio-emocional a temprana }\end{array}$ & $\begin{array}{l}\text { Muestra pequeña } \\
\text { Resultados } \\
\text { afectados por el } \\
\text { enfoque } \\
\text { comunicación de } \\
\text { utilizado o } \\
\text { atribuibles a los más }\end{array}$ \\
\hline
\end{tabular}




\begin{tabular}{|c|c|c|c|c|c|c|}
\hline & & $\begin{array}{l}\text { audífonos } \\
\text { o IC y la } \\
\text { comunicación } \\
\text { oral-auditiva. }\end{array}$ & $\begin{array}{l}\text { Peabody } \\
\text { Goldman-Fristoe } \\
\text { GFTA-2 }\end{array}$ & & $\begin{array}{l}\text { edad en algunos niños con } \\
\text { perdida auditiva } \\
\text { neurosensorial permanente. }\end{array}$ & $\begin{array}{l}\text { sofisticados } \\
\text { tecnológicamente } \\
\text { dispositivos } \\
\text { utilizados. }\end{array}$ \\
\hline $\begin{array}{l}\text { Gran Bretaña } \\
\mathrm{N}^{\circ}=22 \text { niños } \\
\text { sordos y oyentes. } \\
2 \text { grupos: } \\
-5-7 \text { años (sordos } \\
\text { y oyentes) } \\
-11-13 \quad \text { años } \\
\text { (sordos) }\end{array}$ & $\begin{array}{l}\text { Gray, C., Hosie, J., } \\
\text { Russell, P., Scott, } \\
\text { C. Hunter, N. } \\
(2007) \text {. }\end{array}$ & $\begin{array}{lr}\text { Investigar } & \text { el } \\
\text { grado } & \text { de } \\
\text { causalidad que los } \\
\text { niños sordos } \\
\text { hacen de las } \\
\text { emociones. }\end{array}$ & $\begin{array}{l}- \text { Fotografías con } \\
\text { diferentes caras } \\
\text { (felicidad, cólera } \\
\text { tristeza, miedo, } \\
\text { sorpresa ray } \\
\text { repugnancia) } \\
\text {-Historias que } \\
\text { representan } \\
\text { dichas emociones }\end{array}$ & $\begin{array}{l}\text { Diseño comparativo } \\
\text { entre niños sordos de } \\
\text { diferentes edades y } \\
\text { niños oyentes. }\end{array}$ & $\begin{array}{l}\text { No hubo diferencias } \\
\text { significativas entre los niños } \\
\text { sordos y oyentes. } \\
\text { Grupo oyentes: nivel de aciertos } \\
\text { casi tan alto como el grupo de } \\
\text { sujetos sordos mayores. } \\
\text { Error común: interpretar de } \\
\text { forma incorrecta las emociones. }\end{array}$ & $\begin{array}{l}\text { Sujetos } \\
\text { pequeños: } \\
\text { experiencia poca } \\
\text { lengua de signos. } \\
\text { Estudio basado en } \\
\text { pocas emociones. }\end{array}$ \\
\hline $\begin{array}{l}\text { Alemania } \\
\mathrm{N}^{\circ}= \\
\text { participantes. } \\
2 \text { grupos: } \\
-213 \text { niños sordos } \\
-213 \text { padres ( } 95 \% \\
\text { oyentes) }\end{array}$ & $\begin{array}{l}\text { Hintermair, } \quad \text { M. } \\
(2007)\end{array}$ & $\begin{array}{lr}\text { Presentar } & \text { los } \\
\text { problemas } & \\
\text { socioemocionales } \\
\text { frecuentes } & \text { en } \\
\text { niños } & \text { sordos } \\
\text { alemanes } & \text { y } \\
\text { conocer, más } & \text { a } \\
\text { fondo, } & \text { la } \\
\text { veracidad } & \text { del } \\
\text { SDQ } & \end{array}$ & $\begin{array}{l}\text { Cuestionario de } \\
\text { Dificultades y } \\
\text { Fortalezas: SDQ } \\
\text { (Strenghts and } \\
\text { Difficulties } \\
\text { Questionnaire) } \\
\text { para padres y } \\
\text { madres (debían } \\
\text { cubrirlos de } \\
\text { manera } \\
\text { independiente). }\end{array}$ & $\begin{array}{l}\text { Diseño comparativo } \\
\text { entre niños sordos y } \\
\text { oyentes. }\end{array}$ & $\begin{array}{l}\text { La versión alemana del SDQ } \\
\text { puede ser usada para medir los } \\
\text { problemas socioemocionales del } \\
\text { estudiantado. } \\
\text { Predominio de problemas } \\
\text { socioemocionales en sujetos } \\
\text { sordos. }\end{array}$ & $\begin{array}{l}\text { No se incluyeron los } \\
\text { padres de los niños } \\
\text { con sordera en las } \\
\text { escuelas ordinarias } \\
\text { La tasa de respuesta } \\
\text { a la encuesta fue } \\
\text { sólo del } 35,5 \% \\
\text { Muestra pequeña. }\end{array}$ \\
\hline $\begin{array}{l}\text { Florida y } \\
\text { Maryland. } \\
\mathrm{N}^{\circ}=40 \text { sujetos }(8- \\
11 \text { años) } \\
2 \text { grupos: } \\
-20 \text { niños sordos } \\
-20 \text { niños oyentes }\end{array}$ & $\begin{array}{l}\text { Vogel-Walcutt, J.J., } \\
\text { Schatschneider, C., } \\
\text { Bowers, C. (2011) }\end{array}$ & $\begin{array}{l}\text { Determinar las } \\
\text { semejanzas y } \\
\text { diferencias entre } \\
\text { alumnos sordos y } \\
\text { alumnos oyentes. }\end{array}$ & $\begin{array}{l}5 \text { escalas: } \\
\text { Child Rating } \\
\text { Scale (CRS) } \\
\text { Child Behavior } \\
\text { Checklist- } \\
\text { Observation Form } \\
\text { (CBCL-DOF) } \\
\text { Screen for Child } \\
\text { Anxiety Related } \\
\text { Emotional } \\
\text { Disorders }\end{array}$ & $\begin{array}{ll}\text { Diseño } & \text { comparativo } \\
\text { entre } & \text { sordos } y \\
\text { oyentes. } & \end{array}$ & $\begin{array}{l}\text { No existen diferencias } \\
\text { significativas en las habilidades } \\
\text { sociales y emocionales entre } \\
\text { estudiantes sordos y oyentes. } \\
2 \text { diferencias específicas: interés } \\
\text { por la escuela y comportamiento } \\
\text { sobre la tarea. }\end{array}$ & $\begin{array}{l}\text { Muestra pequeña. } \\
\text { Datos no } \\
\text { generalizables dado } \\
\text { que los niños que } \\
\text { utilizan diferentes } \\
\text { métodos de } \\
\text { comunicación. } \\
\text { Participación de sólo } \\
\text { el } 40 \% \text {. }\end{array}$ \\
\hline
\end{tabular}




\begin{tabular}{|c|c|c|c|c|c|c|}
\hline & & & $\begin{array}{l}\text { (SCARED) } \\
\text { Interpersonal } \\
\text { Relationships } \\
\text { Questionnaire } \\
\text { (IRQ } \\
\text { Child Loneliness } \\
\text { Scale (CLS } \\
\end{array}$ & & & \\
\hline $\begin{array}{l}\text { Irlanda } \\
-69 \text { niños con IC } \\
-67 \text { niños oyentes }\end{array}$ & $\begin{array}{lr}\text { Wiefferink, } & \text { C.H., } \\
\text { Rieffe, } & \text { C., } \\
\text { Ketelaar, } & \text { L., } \\
\text { Frijins, J.H. (2012). }\end{array}$ & $\begin{array}{lr}\text { Comparar } & \text { niños } \\
\text { con un IC y niños } \\
\text { con } & \text { audición } \\
\text { normal } & \text { sobre } \\
\text { aspectos } & \text { de } \\
\text { regulación } & \\
\text { emocional } & \text { y } \\
\text { funcionamiento } \\
\text { social y } & \text { la } \\
\text { relación } & \text { entre } \\
\text { ambas. } & \end{array}$ & $\begin{array}{l}\text {-Cuestionarios a } \\
\text { la padres. } \\
\text {-Tareas sencillas }\end{array}$ & $\begin{array}{l}\text { Diseño comparativo } \\
\text { entre sordos (con IC } \\
\text { recientes) y oyentes. }\end{array}$ & $\begin{array}{l}\text {-Los niños oyentes guardan } \\
\text { relación entre la regulación } \\
\text { emocional y el funcionamiento } \\
\text { social. } \\
\text {-Los niños con IC no guardan } \\
\text { dicha relación. } \\
\text {-Las habilidades lingüísticas } \\
\text { están relacionadas con la } \\
\text { competencia social en ambos } \\
\text { grupos. }\end{array}$ & $\begin{array}{l}\text {-Necesidad } \\
\text { desarrollar estudios } \\
\text { longitudinales que } \\
\text { exploren a los niños } \\
\text { con IC, dado que la } \\
\text { mayoría han sido } \\
\text { implantados } \\
\text { recientemente por lo } \\
\text { que existe un menor } \\
\text { número de } \\
\text { experiencias } \\
\text { auditivas en relación } \\
\text { a los sujetos } \\
\text { oyentes. }\end{array}$ \\
\hline $\begin{array}{l}\text { Johor (Malasia) } \\
2 \text { grupos (7-12 } \\
\text { años): } \\
\text { - Sujetos sordo } \\
\text { - Sujetos oyentes }\end{array}$ & $\begin{array}{l}\text { Hanafi, M., Yasin, } \\
\text { M., Bari, S., } \\
\text { Salubin, R. (2012) }\end{array}$ & $\begin{array}{l}\text { Investigar la } \\
\text { inteligencia } \\
\text { emocional }\end{array}$ & Cuestionarios & $\begin{array}{ll}\text { Diseño } & \text { comparativo } \\
\text { entre } & \text { sordos } y \\
\text { oyentes. } & \end{array}$ & $\begin{array}{l}\text { El nivel de } \\
\text { emocional de los sordos está } \\
\text { dentro de la media, aunque la } \\
\text { mayoría tiene habilidades } \\
\text { emocionales bajas. } \\
\text { No hubo diferencias } \\
\text { significativas entre los grupos. }\end{array}$ & Sin especificar \\
\hline $\begin{array}{l}\text { Holanda } \\
\mathrm{N}^{\circ}=26 \text { niños } \\
\text { oyentes y } \\
(11 \text { años) }\end{array}$ & Rieffe, C. (2012) & $\begin{array}{lr}\text { Analizar cómo } \\
\text { comprenden } \\
\text { regulan } \\
\text { emociones } \\
\text { los niños sordos. }\end{array}$ & $\begin{array}{l}\text { Mediante } \\
\text { preguntas- } \\
\text { respuestas: } \\
\text { Tareas: } \\
\text { - Identificación de } \\
\text { las emociones } \\
\text { básicas: alegría, }\end{array}$ & $\begin{array}{l}\text { Diseño comparativo } \\
\text { entre sujetos sordos y } \\
\text { oyentes. }\end{array}$ & $\begin{array}{l}\text { Sujetos oyentes: identifican y } \\
\text { gestionan tanto unas como otras. } \\
\text { Sujetos sordos demuestran } \\
\text { menos respuestas empáticas. }\end{array}$ & $\begin{array}{l}\text { Resultados no } \\
\text { generalizables } \\
\text { (muestra pequeña). }\end{array}$ \\
\hline
\end{tabular}




\begin{tabular}{|c|c|c|c|c|c|c|}
\hline & & & $\begin{array}{l}\text { tristeza, ira y } \\
\text { miedo. } \\
\text { - Identificación de } \\
\text { múltiples } \\
\text { emociones. }\end{array}$ & & & \\
\hline $\begin{array}{l}\text { Tehran (Irán). } \\
\mathrm{N}^{\circ}=40 \\
\text { estudiantes } \\
\text { sordos } \\
\text { años) }\end{array}$ & $\begin{array}{l}\text { Pourmohamadreza, } \\
\text { M., Ashori, M., \& } \\
\text { Jalilabkenar, S. } \\
\text { (2013). }\end{array}$ & $\begin{array}{lr}\text { Demostrar } & \text { la } \\
\text { importancia } & \text { de } \\
\text { desarrollar } & \\
\text { programas } & \\
\text { educativos } & \text { que } \\
\text { avalen } & \text { la } \\
\text { educación } & \\
\text { emocional } & \text { en } \\
\text { estudiantes sordos }\end{array}$ & $\begin{array}{l}\text { 1.Test de } \\
\text { inteligencia } \\
\text { Weschsler } \\
\text { (WISC-R) } \\
\text { 2. Cuestionario de } \\
\text { Salud General } \\
\text { (General Health } \\
\text { Questionnaire) }\end{array}$ & $\begin{array}{l}\text { Estudio } \\
\text { semiexperimental } \\
\text { con diseño preprueba } \\
\text { y postprueba: } \\
\text { Programa de IE }\end{array}$ & $\begin{array}{l}\text { La inteligencia emocional que se } \\
\text { entrena mejora y tiene efectos } \\
\text { positivos para la salud mental } \\
\text { Reducción de la situación de } \\
\text { ansiedad y depresión. } \\
\text { Educar a las personas sordas a } \\
\text { través de las emociones evita } \\
\text { problemas de ansiedad, } \\
\text { depresión y ayuda a regular y } \\
\text { controlar las propias emociones. }\end{array}$ & Muestra pequeña \\
\hline $\begin{array}{l}\text { Israel } \\
\mathrm{N}^{\circ}=53 \text { sujetos. } \\
3 \text { grupos: } \\
-20 \text { con IC } \\
-10 \text { niños sordos } \\
-\quad 23 \text { niños } \\
\text { oyentes }\end{array}$ & $\begin{array}{l}\text { Ziv, M., Most. T., } \\
\text { Cohen, S. (2013). }\end{array}$ & 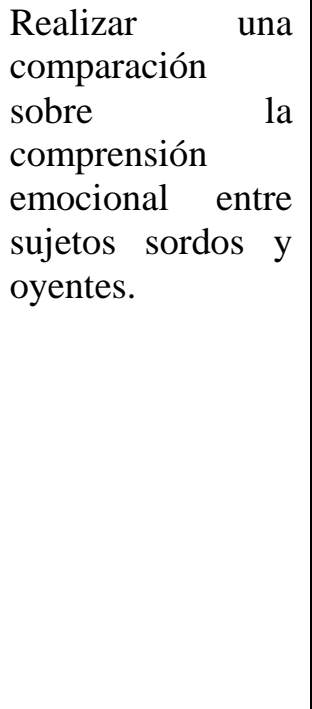 & $\begin{array}{l}\text {-Una medida de } \\
\text { lengua: } \\
\text { (PPVT-R) } \\
\text {-Comprensión de } \\
\text { emociones: } \\
\text { 1. Identificación } \\
\text { de las caras } \\
\text { 2. Comprensión } \\
\text { de las emociones } \\
\text { en contextos } \\
\text { típicos } \\
3 \text {. Perspectiva } \\
\text { afectiva } \\
\text { historias de } \\
\text {-Teoría de la } \\
\text { Mente }\end{array}$ & $\begin{array}{l}\text { Diseño comparativo } \\
\text { entre sordos y/o con } \\
\text { IC y oyentes. }\end{array}$ & 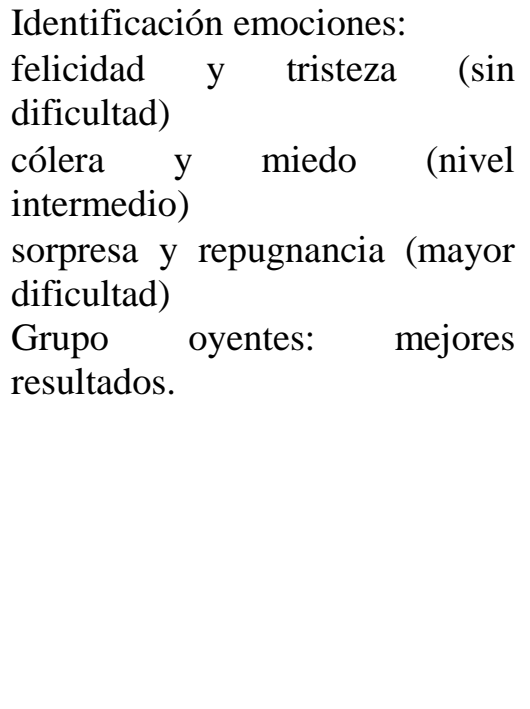 & $\begin{array}{lr}\text { No } & \text { examina } \\
\text { aspectos } & \text { cognitivos } \\
\text { sociales. } & \end{array}$ \\
\hline
\end{tabular}




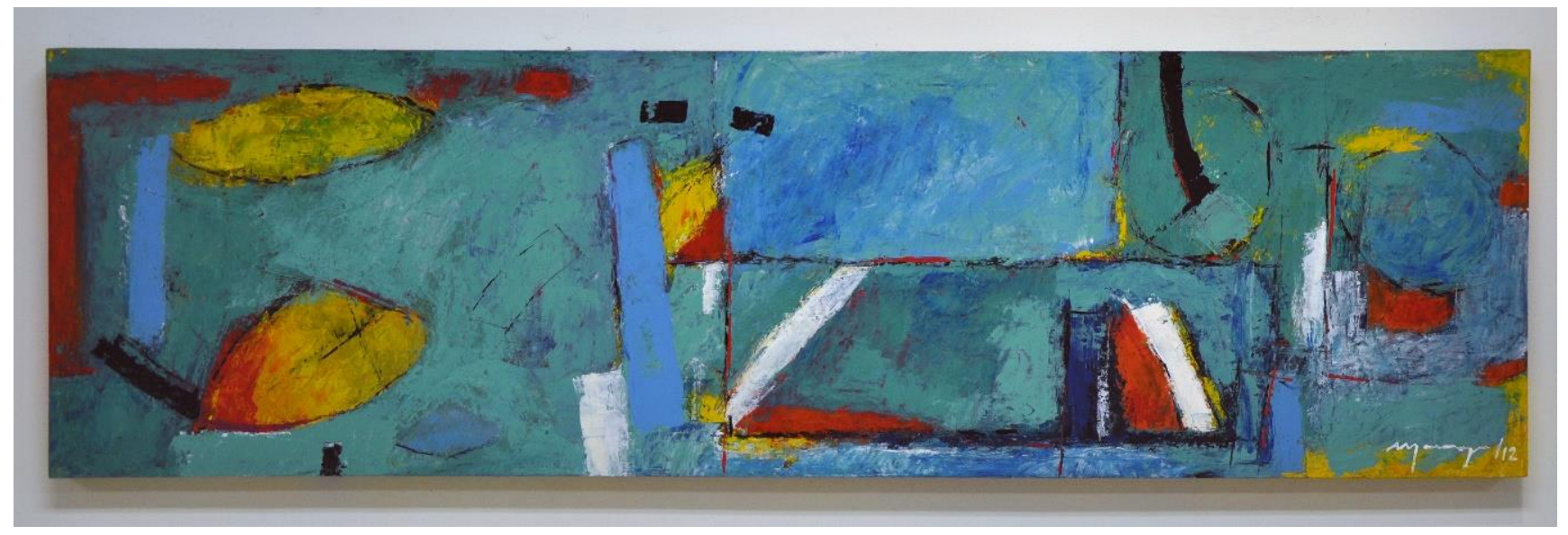

Título de la obra: Señales

Técnica: pigmentos industriales sobre polietileno

Dimensiones de la obra: 140 c.ms $x 40$ c.ms

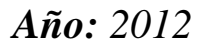

Nombre de la imagen adjunta: 007 